\title{
Distance learning: The "Extension for Community Healthcare Outcomes: ECHO" project: A case study
}

\author{
Jularat Panyayen MD, Teerapat Nantsupawat MD, Wittawat Tangwijitsakul MD, \\ Nopakoon Nantsupawat MD
}

\begin{abstract}
This article reports a case conference that was part of the Extension for Community Healthcare Outcomes $\left(\mathrm{ECHO}^{\circledR}\right)$ project, which is a model of monthly telemedicine conferences on chronic disease and behavioral health, important topics for primary care teams in rural areas and for university-based specialists. The $\mathrm{ECHO}^{\circledR}$ project has proved to be a successful learning model in health care. The main goal of the project is moving knowledge not the patients, so this tele-mentoring builds capacity and creates access to high-quality specialty care serving local communities. A secondary goal is shared learning between community providers and specialists about best practices that are practical, achievable, and sustainable for the community. We present the case of a critical care patient who was brought to the emergency department with left-side weakness and dysarthria. Computed tomography of the head and electrocardiograms established the diagnosis during the admission. Discussion points from a multidisciplinary team and specialty consultants via telemedicine are listed in this article.
\end{abstract}

Keywords: telemedicine, teleconsulting, primary care, developing countries, rural area

\section{INTRODUCTION}

Thailand is a developing country in which there are some limitations of medical practice in rural areas. Distribution of the health care resources in rural areas is less than in urban areas. Furthermore, access to specialized care is more difficult due to geography, in which distance can be an important factor. ${ }^{1,2}$ Telemedicine is one of the solutions to this problem; however, although it is currently deployed in Thailand, telemedicine is still not widely used in primary care, especially in rural areas. The availability and accessibility of telemedicine usually vary depending on expertise, experience, technology resources, and hospital policy in each rural area. ${ }^{3-5}$

The Faculty of Medicine at Chiang Mai University $(\mathrm{CMU})$ is a tertiary care center and referral center

Corresponding author: Nopakoon Nantsupawat Contact Information: Nopakoon.n@cmu.ac.th

DOI: 10.12746/swrccc.v9i40.889 serving a subdistrict in Chiang Mai and 16 other provinces in the Northern region of Thailand. Since 2018, the Southeast Asia HIV Addiction Technology Transfer Center (SEA-HATTC) at the Faculty of Medicine at $\mathrm{CMU}$ has partnered with the Missouri Telehealth Network and the University of Missouri School of Medicine (Columbia, Missouri, USA) to use the $\mathrm{ECHO}^{\circledR}$ (Extension for Community Healthcare Outcomes) model to present regular monthly telemedical conferences on chronic disease and behavioral health, important topics for doctors, family physicians from remote areas, nurses, multidisciplinary care providers, medical school lecturers, and medical students. The $\mathrm{ECHO}^{\circledR}$ project is one of several widely used, collaborative models of medical education and clinical management. It has proved to be a successful learning model in health care for communities and covers numerous chronic conditions, with 162 hubs based in the United States and with 324 hubs across 37 countries. ${ }^{6,7}$ The number of participants ranges from 40 to 60 in each session and often involves more than eight different disciplines in health care. 
This article presents a case conference that was part of the $\mathrm{ECHO}^{\circledR}$ project; this conference helped primary care physicians and multidisciplinary teams in clinical decision-making and management of some challenging points and pitfalls in primary care.

\section{CAse study}

A 61-year-old woman presented with acute drowsiness, worsening left-sided weakness, and dysarthria three hours prior to admission. She complained of vertigo and vomited four times while she was sitting in her living room. She then could not lift her left leg and had slurred speech. Her relatives brought her to the rural hospital which is $44.3 \mathrm{~km}$ from her house and $79.2 \mathrm{~km}$ from the nearest tertiary care center.

Her past medical history included hypertension, dyslipidemia, ischemic strokes, epilepsy, and atrial fibrillation diagnosed at age 44 years old. After the first ischemic stroke, she had motor power grades 0 and 4 in her left upper and left lower extremity, respectively. For baseline functional status, she was able to bathe, dress, and prepare meals with assistance, and walk with a three-point cane. Medications were aspirin (81 $\mathrm{mg})$ one tablet once daily, phenobarbital $(60 \mathrm{mg})$ one tablet before bedtime, amlodipine $(5 \mathrm{mg}$ ) one tablet once daily, enalapril $(5 \mathrm{mg})$ one tablet once daily, digoxin $(0.25 \mathrm{mg})$ half tablet once daily, and simvastatin (40 mg) one tab before bedtime.

Vitals signs included blood pressure $157 / 100 \mathrm{mmHg}$, pulse rate 49 beats per minute, respiratory rate 18 breaths per minute, and temperature $37.0^{\circ} \mathrm{C}$. Room air oxygen saturation was $99 \%$. Her heart rhythm was irregular, S1 and S2 were normal, and there were no murmurs. Lung sounds were clear and equal. Neurological examinations were E4V2M5, pupils in both eyes $3 \mathrm{~mm}$ reactive to light, full extraocular movement, no nystagmus, left facial palsy (upper motor neuron lesion), and dysarthria. Normal right-sided motor power. Motor power grade 0 left upper extremity and grade 2 (worsened) left lower extremity.

Complete blood count and basic metabolic panel were within normal limits. Fasting blood glucose was $110 \mathrm{mg} / \mathrm{dL}$. Lipid profile revealed total cholesterol $186 \mathrm{mg} / \mathrm{dL}$, triglyceride $141 \mathrm{mg} / \mathrm{dL}, \mathrm{HDL} 51 \mathrm{mg} / \mathrm{dL}$, and LDL $133 \mathrm{mg} / \mathrm{dL}$.
Brain computed tomography demonstrated an old infarction of right fronto-parietal-temporal lobes, left occipital lobes, left frontal cortex, right insular cortex, right basal ganglia, and right corona radiata (Figure 1). Chest $x$-ray showed mild cardiomegaly without infiltrates or pleural effusion. Electrocardiogram (ECG) showed atrial fibrillation with slow ventricular response 42/min, corrected QT 466 milliseconds. There were reversed check mark ST-T changes consistent with digoxin effect (Figure 2).

She was treated as symptomatic bradycardia with atropine $0.6 \mathrm{mg}$ intravenously. Following that administration, her heart rate increased to 73 beats per minute with blood pressure of $147 / 93 \mathrm{mmHg}$. Glasgow coma scale improved to E4V5M6. The patient was discharged from the hospital on day 3 . The primary care physician at the rural hospital then presented this case to project $\mathrm{ECHO}^{\circledR}$ for further evaluation and management.

\section{DISCUSSION POINTS FROM THE MULTIDISCIPLINARY TEAM AND SPECIALTY CONSULTANTS VIA TELEMEDICINE}

1. Atrial fibrillation was diagnosed at the time of her first ischemic stroke. Her $\mathrm{CHA}_{2} \mathrm{DS}_{2}$ VASc score was 4. Her HAS-BLED score was 2. Brain imaging was consistent with cardioembolic stroke. With the limitation of specialty consultants in rural hospitals, undertreatment of oral anticoagulation (OAC) can lead to recurrent ischemic strokes that might have been prevented. However, starting OAC now still provides benefit in preventing future stroke. The cost of non-vitamin $\mathrm{K}$ antagonists is not covered by the universal health insurance in Thailand, so warfarin with a goal INR of $2-3$ was suggested. ${ }^{8}$

2. Long term digoxin treatment, especially in the elderly, may increase risk of toxicity due to altered pharmacokinetics and the narrow therapeutic index of digoxin. Symptoms of digoxin toxicity may include anorexia, cognitive changes, hazy vision, and bradyarrhythmias. ${ }^{9}$ Therefore, we recommended discontinuing digoxin and considering a beta-blocker instead.

3. Improper statins were prescribed. With the Thai estimated 10 -year cardiovascular risk of $10.16 \%$ 

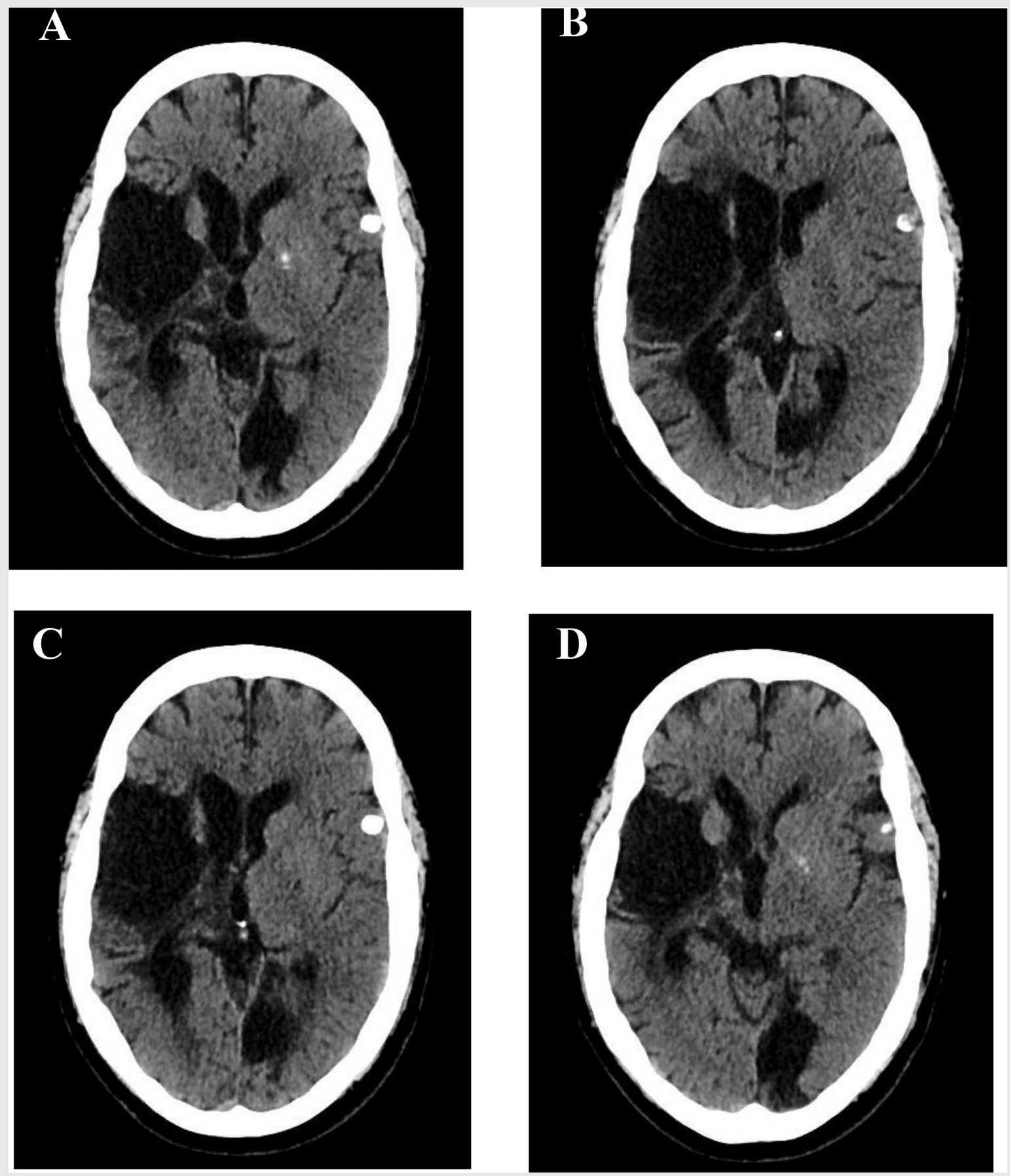

Figure 1. Brain computed tomography demonstrated old infarction of right fronto-parietal-temporal lobes, left occipital lobes, left frontal cortex, right insular cortex, right basal ganglia, and right corona radiata. (label A to D, from caudal section to occipital section) 


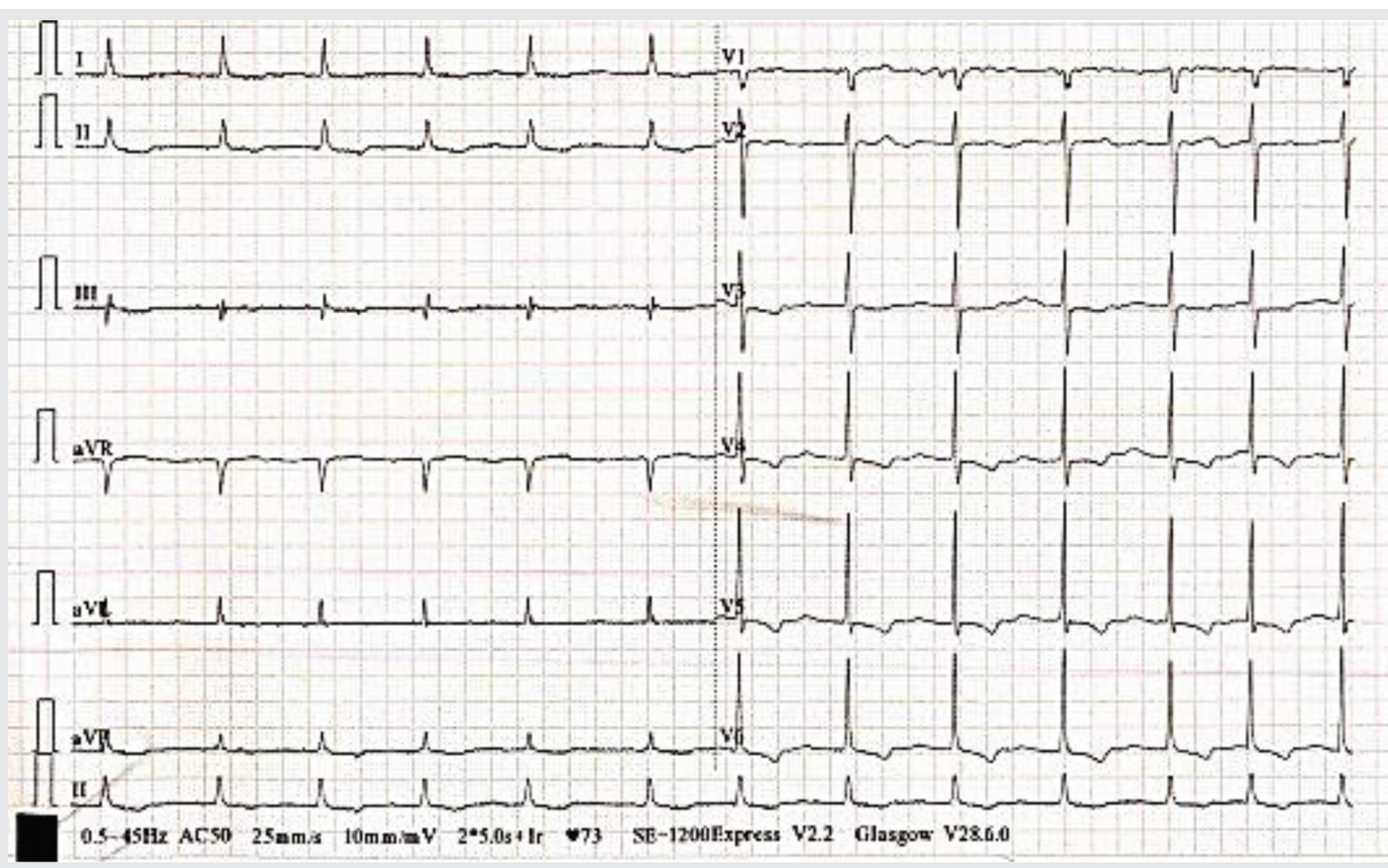

Figure 2. Electrocardiogram showed atrial fibrillation with slow ventricular rate at 42 per minute and a corrected QT 466 milliseconds. Reversed check mark ST-T changes were noticed in V2-V6.

and established atherosclerotic cardiovascular disease, the patient should be given a high-potency statin (atorvastatin $40 \mathrm{mg}$ or rosuvastatin $20 \mathrm{mg}$ ) with an LDL goal of $<70 \mathrm{mg} / \mathrm{dL}$ or at least to a $50 \%$ reduction from baseline LDL.

4. Recommended additional investigations and monitoring include:

A. Transthoracic echocardiography should be performed as initial work up for atrial fibrillation to evaluate for ventricular systolic and diastolic function, atrial size, and valvular abnormalities. The local team helped arrange an echocardiology study at the echocardiographic facility.

$B$. The clinic staff should monitor the patient for side effects and adverse events, such as falls, bleeding risk, daily function/deconditioning, unilateral neglect, spastic gait, and other psychosocial outcomes like depression and caregiver burden within the family.

\section{Conclusion}

Providing primary care to patients with critical illnesses is a challenging task that requires highly skilled and experienced practitioners. Undertreatment and under investigation are the most common pitfalls in primary care; thus, experts and multidisciplinary health teams should collaborate to provide optimal care of these patients.

Project $\mathrm{ECHO}^{\circledR}$ is a model of a teleconference that shares case-based learning about clinical management with a multidisciplinary team and university-based specialists. This conference is different from the two-sided telemedicine approach; it increases the capacity of care 
for all physicians, multidisciplinary care providers, and other attendants who attend the conference. As a result, the primary care team can provide comprehensive, best-practice care to patients with complex health conditions, right where they live. Furthermore, this project also provides other benefits, e.g., it reduces the need for patient/caregiver transportation and reduces the number of patients at the referral center..$^{10}$ In our setting, we could observe an engagement from the audience during the discussion in the conference, other practitioners shared their experiences and asked questions of the entire group, and we received positive feedback from the providers' team and other participants. Even though, in a developing country like Thailand, more systematically and comprehensively evaluation of the outcomes should be done to establish internally and externally validity and to demonstrate the value of this model.

Article citation: Panyayen J, Nantsupawat T, Tangwijitsakul W, Nantsupawat N. The Southwest Respiratory and Critical Care Chronicles 2021;9(40):60-64

From: Primary Care Department (JP, WT), Lamphun Hospital, Lamphun, Thailand; Internal Medicine Department (TN), Faculty of Medicine, Chiang Mai University, Chiang Mai, Thailand; Family Medicine Department (NN), Faculty of Medicine, Chiang Mai University, Chiang Mai, Thailand

Submitted: $6 / 22 / 2021$

Accepted: $7 / 3 / 2021$

Reviewer: Drew Payne DO

Conflicts of interest: none

This work is licensed under a Creative Commons Attribution-ShareAlike 4.0 International License.

\section{REFERENCES}

1. Combi C, Pozzani G, Pozzi G. Telemedicine for developing countries. a survey and some design issues. Appl Clin Inform 2016;7(4):1025-50.

2. Pagaiya N, Kongkam L, Sriratana S. Rural retention of doctors graduating from the rural medical education project to increase rural doctors in Thailand: a cohort study. Hum Resour Health 2015;13:10.

3. Latifi R, Dasho E, Shatri Z, et al. Telemedicine as an innovative model for rebuilding medical systems in developing countries through multipartnership collaboration: the case of Albania. Telemed J E Health 2015;21(6):503-9.

4. Ikhu-Omoregbe NA, Ayo CK, Ehikioya SA. A deployable framework for mobile telemedicine applications. Stud Health Technol Inform 2006;121:36-41.

5. Nittari G, Khuman R, Baldoni S, et al. Telemedicine practice: review of the current ethical and legal challenges. Telemed J E Health 2020;26(12):1427-37.

6. Spaulding R, Henley W, Wright S, et al. Project ECHO, communities of practice, and a successful opioid reduction outcome. Arch Community Med Public Health 2020;6(1): 074-076. DOI: https://dx.doi.org/10.17352/2455-5479.000081.

7. Spaulding R, Henley W, Lyon J. Project ECHO in Kansas: Qualitative assessment of participants' of self-efficacy, knowledge, translation, and rural-urban comparisons. MetaECHO Conference 2019, New Mexico, USA.

8. Mitrani LR, De Los Santos J, Driggin E, et al. Anticoagulation with warfarin compared to novel oral anticoagulants for atrial fibrillation in adults with transthyretin cardiac amyloidosis: comparison of thromboembolic events and major bleeding. Amyloid. 2021;28(1):30-4.

9. Vyas A, Bachani N, Thakur H, et al. Digitalis toxicity: ECG vignette. Indian Heart J. 2016;68 Suppl 2:S223-S5.

10. Di Cerbo A, Morales-Medina JC, Palmieri B, et al. Narrative review of telemedicine consultation in medical practice. Patient Prefer Adherence. 2015;9:65-75. 